\title{
Chapter 4 \\ The Role of Liquid Biopsies for Monitoring Disease Evolution
}

\author{
Ingeborg Tinhofer
}

\section{Introduction}

Tissue biopsies have been used by clinicians to diagnose and manage disease for more than 1000 years [1]. The first report on the use of needles for puncturing a thyroid gland cancer came from a court physician to the Andalusian caliph Al-Hakim II [1]. From then until the modern era of precision oncology, tissue biopsies have remained the most widely used tool not only for cancer detection and staging but also for molecular tumor profiling to guide targeted therapy for the individual patient. However, tumor biopsies generally involve invasive medical procedures that can be difficult and risky, especially in cancer patients with advanced disease. Moreover, even if a fresh tumor biopsy can be safely taken, the material for the molecular analysis might be limited as a relevant amount of the tissue is reserved for routine pathology. Due to the restrictions of solid tissue sampling, it is often necessary to resort for molecular profiling to archival tumor samples that were collected long time prior to the planned molecular analysis, typically at the time of initial biopsy or surgical resection. Changes in the mutational pattern and/or subclonal spectrum of tumors occurring during disease progression can decrease the diagnostic accuracy of the molecular test in this situation. The invasive nature of solid tissue collection

\footnotetext{
I. Tinhofer $(\bowtie)$

Department of Radiooncology and Radiotherapy, Charité - Universitätsmedizin Berlin, Corporate Member of Freie Universität Berlin, Humboldt-Universität zu Berlin, and Berlin Institute of Health, Berlin, Germany

German Cancer Research Center (DKFZ), German Cancer Consortium (DKTK) Partner Site Berlin, Berlin, Germany

German Cancer Research Center (DKFZ), Heidelberg, Germany

German Cancer Consortium (DKTK), Berlin, Germany

e-mail: ingeborg.tinhofer@charite.de
} 
Table 4.1 Cell-free tumor DNA versus solid tissue biopsies: pros and cons

\begin{tabular}{|c|c|c|c|c|}
\hline Consideration & & Cell-free tumor DNA & & Solid tissue \\
\hline Sampling & $\begin{array}{l}+ \\
+\end{array}$ & $\begin{array}{l}\text { Non or minimally invasive } \\
+ \text { Serial monitoring easy }\end{array}$ & - & $\begin{array}{l}\text { Invasive, more challenging to obtain } \\
\text { Serial testing more difficult }\end{array}$ \\
\hline \multirow[t]{2}{*}{ Biology } & - & $\begin{array}{l}\text { No direct correlation with } \\
\text { tumor histology or cellular } \\
\text { phenotype possible }\end{array}$ & + & $\begin{array}{l}\text { Can correlate with histology and } \\
\text { cellular phenotype }\end{array}$ \\
\hline & + & $\begin{array}{l}\text { Allows global view on } \\
\text { intratumoral heterogeneity }\end{array}$ & - & Represents one small tumor region \\
\hline \multirow[t]{3}{*}{ Pre-analytical } & + & $\begin{array}{l}\text { Easy to standardize across } \\
\text { centers }\end{array}$ & + & $\begin{array}{l}\text { Uses existing, validated tissue } \\
\text { processing and handling approaches }\end{array}$ \\
\hline & - & $\begin{array}{l}\text { Need for specific blood } \\
\text { stabilization tubes }\end{array}$ & & \\
\hline & - & $\begin{array}{l}\text { Confounding patient-related } \\
\text { factors poorly characterized } \\
\text { (clonal hematopoiesis) }\end{array}$ & & \\
\hline Clinical utility & - & $\begin{array}{l}\text { Limited evidence for } \\
\text { treatment selection and } \\
\text { screening }\end{array}$ & + & $\begin{array}{l}\text { Substantial evidence for treatment } \\
\text { selection in multiple entities for early } \\
\text { and advanced cancers }\end{array}$ \\
\hline
\end{tabular}

makes it also very difficult to accomplish serial sampling under treatment, which would be required for the analysis of clonal evolution and acquired drug resistance.

The above-mentioned hurdles can be overcome by liquid biopsies which can harvest cancer-related biomarkers from blood, saliva or urine. Advantages and disadvantages of liquid and solid biopsies are summarized in Table 4.1. Liquid biopsies represent a non- or minimally invasive, inexpensive source for tumor material, including circulating tumor cells (CTCs) and tumor-derived cellular components like extracellular vesicles, miRNA, protein, and cell-free (cf) circulating tumor (ct) DNA. Liquid biopsies allow 'real time' assessment of the tumor status, thereby providing a global view on spatial and temporal intratumoral heterogeneity both in primary and metastatic disease. Liquid biopsies have successfully been used for selection of molecular treatment [2] as well as in-depth analysis of the molecular changes associated with acquired drug resistance [3]. While serial tissue biopsies for monitoring depth and duration of treatment responses are rarely possible, liquid biopsies can be applied for this purpose, allowing detection of tumor progression up to several months before clinical relapse [4]. High sensitivity at stages of very low tumor burden would also give the chance to use liquid biopsies for early cancer screening. In fact, laboratories around the world are currently competing by developing cancer screening tests based on a simple blood sample.

In this article, I will summarize current evidence of the diagnostic value of liquid biopsies for disease monitoring in HNSCC, with special emphasis on the potential clinical value in cancer screening, post-treatment surveillance, molecular profiling for molecularly guided treatment selection, monitoring of treatment efficacy and the analysis of acquired drug resistance. 


\section{Liquid Biopsies for Cancer Screening and Disease Monitoring}

The concept of using a blood test for cancer screening is not new. Blood tests for prostate specific antigen (PSA) or cancer antigen 125 (CA-125) have been broadly used for early detection of prostate or ovarian cancer. However, these previous methods can be extremely nonspecific and result in high rates of false positive tests. A blood test for large-scale population screening would require high specificity, clinically useful sensitivity, and highly accurate identification of the tissue of origin, in order to limit costs and the complexity of evaluating asymptomatic patients. A fundamental question for recent DNA-based approaches is whether small tumors would release sufficient amounts of tumor DNA into the circulation to allow sensitive detection of the cancer-associated changes.

\section{Early Detection of Virally Associated HNSCC Based on Plasma DNA}

First evidence for a potential application of analysis of circulating tumor-related DNA for screening for HNSCC came from Epstein-Barr virus (EBV)-associated undifferentiated nasopharyngeal cancer. Earlier observations of short EBV DNA fragments in blood samples from nasopharyngeal carcinoma patients [5], which were released by carcinoma cells and not associated with viral particles [6], had suggested that plasma EBV DNA might represent a useful biomarker for identifying early-stage nasopharyngeal carcinoma among asymptomatic individuals. This hypothesis was successfully tested in a large Asian screening study enrolling more than 20,000 participants [7]. The investigators could confirm that detection of viral DNA in plasma by real-time polymerase-chain reaction (PCR) can identify individuals with early-stage disease with sensitivity and negative predictive values of $97 \%$ and $99.995 \%$, respectively. Circulating viral DNA in human papilloma virus (HPV)-driven oropharyngeal carcinoma has emerged as further promising biomarker for screening and disease monitoring in HNSCC because approximately $90 \%$ of patients have detectable plasma HPV DNA at the time of diagnosis $[8,9]$. It was also shown that kinetics analysis of HPV DNA can be used to predict the likelihood of disease control after definitive chemoradiation [10]. In the latter study, having high baseline copy number ( $>200$ copies $/ \mathrm{mL}$ ) and $>95 \%$ clearance of HPV DNA by day 28 of chemoradiation was established as a favorable clearance profile associated with improved outcome [10]. Future clinical trials are certainly necessary to explore whether earlier detection of cancer relapse also improves postrecurrence survival outcomes. If so, then integration of HPV DNA-based monitoring might support the current worldwide efforts of developing de-escalated treatment strategies for HPV-positive oropharyngeal carcinoma patients. 


\section{Cell-free DNA Analysis in Non-virally Related HNSCC}

In non-virally related HNSCC, most attempts of developing a non-invasive test for screening and disease monitoring have failed so far. The large interpatient heterogeneity observed in genomic profiles from these tumors and the absence of recurrent hotspot driver alterations have hampered the development of a broadly applicable screening tool based on a single biomarker. Point mutations in the tumor suppressor gene TP53 represent the most frequent genetic alteration in HNSCC [11], suggesting that TP53 mutant variants might represent a promising biomarker for screening and disease monitoring in HPV-negative carcinomas. In line with this assumption, previous molecular studies have identified the presence of mutant TP53 variants in histologically clear surgical margins as potential marker of residual disease identifying patients at high risk of tumor recurrence $[12,13]$. Detection of tumor specific TP53 mutations in plasma cfDNA from HNSCC patients using digital droplet PCR was shown to be technically feasible, providing further support for the use of TP53 alterations as diagnostic biomarker in post-treatment surveillance of HNSCC patients [14]. However, the use of cfDNA-based approaches interrogating singlenucleotide variants that focus on key gene alterations such as TP53 might be less useful for blood-based cancer screening, as this approach may be hampered by confounding signals from clonal hematopoiesis associated with blood-specific mutations in cancer-associated genes like TP53 [15]. Similarly, approaches based on detecting copy number alterations e.g. in genes at the chromosome 11q13 locus displaying amplifications in approximately one third of HPV-negative HNSCC patients [11] may be limited by smaller relative differences between cases and controls, resulting in a need for increased sequencing depth as well as technical variation restricting the signal-to-noise ratio [16].

\section{DNA Methylation Analysis for Cancer Screening}

Recently, the Circulating Cell-free Genome Atlas (CCGA) consortium [17] has launched a large prospective, observational, longitudinal, case-control study for discovery, training, and validation of a multi-cancer screening test. Based on bisulfite sequencing of plasma cfDNA and using machine learning algorithms, a classifier was developed and validated for cancer detection and tissue of origin localization [17]. Recently, very promising results were reported from a pre-specified CCGA sub-study including 6689 participants with previously untreated cancer $(n=2482)$ or without cancer $(n=4207)$ [18]. cfDNA sequencing of informative methylation patterns detected a broad range of cancer types at metastatic and non-metastatic stages with specificity and sensitivity performance approaching the goal for population-level screening [18]. Although good sensitivity (i.e. $>85 \%$ over all stages) at a fixed test specificity of $>99.8 \%$ was observed in the subset of HNSCC cases, results have to be interpreted cautiously due to low HNSCC patient numbers 
both in the training $(n=65)$ and validation set $(n=18)$ of this study [18]. Interestingly, the investigators found that incorrect tissue of origin identification by the methylation classifier often occurred among HPV-driven cancers (e.g. cervix, anus, head and neck cancers), suggesting that test accuracy might be further improved by leveraging this information.

\section{Cell-Free Circulating Tumor DNA for Mutation Profiling}

A mutational load ranking in the upper third of all tumor entities [19] and large interpatient genetic heterogeneity [11] are key features of HNSCC. Signs of high genetic instability are primarily detected in cases with a history of heavy smoking and alcohol consumption, most likely resulting from the extensive DNA damage that has been caused by tobacco carcinogen exposure for years. Exacerbating the complexity of the genetic landscape in HNSCC, intratumoral heterogeneity in terms of spatial and temporal differences in the mutational patterns of key driver genes can occur [20-22]. First evidence of ctDNA being a suitable source for studying the mutational landscape of tumors was provided by the landmark study of Bettegowda and colleagues in which 640 patients with various cancers were included [23]. The investigators were able to demonstrate that mutant DNA fragments can be found at relatively high concentrations in the blood circulation of most patients with metastatic cancer and at lower but detectable concentrations in a substantial fraction of patients with localized disease [23]. In the small subgroup of HNSCC patients $(\mathrm{n}=12)$ included in this study, mutant ctDNA was detected in $70 \%$ of cases [23]. One of the largest subsequent studies so far including 25,578 blood specimens from 21,807 patients with over 50 different cancer types confirmed that mutations in genes associated with cancer can be identified in circulating plasma DNA in the vast majority of patients with advanced cancer [24]. Schwaederle et al. examined the frequency of genetic mutations of ctDNA in 670 cancer patients, of whom 25 had HNSCC, and reported that HNSCC harbors the highest frequency of ctDNA mutations in plasma when compared to lung, gastrointestinal, brain, and breast cancers [25].

The preliminary results from the small HNSCC cohorts included in these histology-agnostic studies were corroborated by a study specifically focusing on HNSCC patients $(\mathrm{n}=93)$ in whom mutations (mainly affecting TP53 in HPVnegative and PIK3CA in HPV-positive cases) were detected in $81 \%$ and $85 \%$ of plasma and saliva samples, respectively [26]. Recently, Galot and coworkers specifically explored the relevance of plasma ctDNA to characterize the mutational landscape in recurrent/metastatic HNSCC [27]. Using a panel of 604 cancer-related genes they reported mutant variant detection in $20 / 39$ patients (51\%). In line with the above mentioned studies across different histologies, a significantly higher probability for ctDNA detection was observed in patients with metastatic disease compared to patients with only locoregional recurrence (70\% vs. 30\%) [27]. This finding suggests a potential limitation of panel NGS-based ctDNA analysis in R/M 
HNSCC given that around one third of recurrent HNSCC patients will have locoregional relapse without distant metastases. However, the lower detection rate of mutant variants in locoregional recurrence in the study of Galot et al. could also have technical rather than biological reasons. Indeed, by applying the more sensitive digital droplet PCR the detection rates could be significantly increased [27].

\section{Concordance Between Liquid and Solid Tissue-Based Mutational Analysis}

In the large observational study of Zill et al. the commercially available Guardant 360 assay (GuardantHealth Inc., Redwood City, CA) covering approximately 70 actionable tumor mutations was used [24]. It was shown that ctDNA mutation patterns were highly consistent with the distribution reported for tumor tissue in the publicly available The Cancer Genome Atlas (TCGA), with correlations ranging from 0.90 to 0.99 [24]. Comparative analysis using matched archival tissue in a subset of 386 patients confirmed the overall high concordance in sequencing results of liquid and solid tumor biopsies [24]. Of note, test accuracy of ctDNA sequencing increased to $98 \%$ when blood and tumor tissue were collected less than 6 months apart. In contrast to these promising results, a remarkably poor overall concordance between molecular profiles established from liquid and solid tumor biopsies was reported by Galot and colleagues in R/M HNSCC [27]. Considering the 18 patients from whom blood and tissue samples were available, only $19 \%$ of the mutant variants (40/209) identified in solid tumors were also detected in plasma. A similar observation was made in a small study of HNSCC cases $(\mathrm{n}=36)$ harboring mutations in either TP53, NOTCH1, CDKN2A, CASP8 or PTEN in tumor tissue, of which only $28 \%$ could be detected in plasma cfDNA [28].

Currently used NGS panels for ctDNA analysis range from small panels of 20 genes to large comprehensive panels of up to several hundred genes. It is very likely that the above-described differences in variant detection between tumor tissue sequencing and cfDNA sequencing depend on the used NGS technology and platform. Most targeted NGS panels originally developed for tissue sequencing have an average sequencing depth of 500×. This coverage has shown to be sufficient to give consistent results in the detection of single nucleotide variants (SNVs) and small insertion/deletions (indels) in tumor tissues [29]. Given the low allele fractions of mutant variants (median: $0.41 \%$ ) in plasma samples in the majority of cancer patients [24], a higher sequencing depth will be required for sensitive mutation detection in plasma cfDNA. Since coverage is usually inversely proportional to the number of genes to be sequenced, an increase in sensitivity of ctDNA-based mutational profiling might thus be realized by using small sets of genes harboring known actionable alterations rather than comprehensive panels of several hundred genes. Indeed, ultra-high sequencing depths (i.e. 50,000-100,000× coverage) combined with a molecular barcoding strategy and in silico elimination of highly stereotypical 
background artifacts were shown to significantly improve recovery of ctDNA molecules, allowing detection of mutant variants down to allele frequencies of $0.004 \%$ [30-32]. This high sensitivity however comes at the price of significantly higher costs per single analysis, raising concerns about affordability in clinical routine, especially if serial liquid biopsy analyses might become part of the routine followup scheme for cancer patients.

\section{ctDNA Versus CTCs: Which Is the Better Source for Mutation Analysis?}

To our knowledge, a comparative analysis of whether ctDNA or CTCs might represent the better source for genomic molecular profiling of HNSCC tumors, at situations when tumor tissue collection is not feasible, is missing until now. First evidence from lung cancer suggested superiority of CTC-over plasma ctDNA-based analysis, since EGFR activating mutation were detected in CTCs from 11 of 12 patients (92\%) but only in matched plasma ctDNA from 4 of 12 patients $(33 \%)(\mathrm{P}=0.009)$ [33]. In contrast, mutation detection at comparable frequencies was reported for CTC-derived genomic material and paired plasma ctDNA from studies in breast [34] and colon cancer [35]. In a relevant number of cases though, CTCs exhibited a mutation that was not detected in ctDNA, and vice versa [35]. Mutation detection in CTCs and plasma-ctDNA might thus provide complementary information suggesting the use of an integrated liquid biopsy approach $[34,35]$.

\section{Liquid Biopsies for Treatment Selection and the Analysis of Resistance Mechanisms}

Perhaps most importantly, evidence is accumulating that liquid biopsies can be used to predict drug response and drug resistance in patients initiating a targeted therapy, pointing to their potential in precision medicine. Of clinical relevance, taking into account FDA-approved agents and eligibility for clinical trials, the ctDNA assay used in the study of Schwaederle et al. identified a possible treatment option for approximately one half of all patients [25]. Furthermore, nearly 1 in $4 \mathrm{ctDNA}$ alteration-positive patients (23\%) across 6 cancer indications in the study of Zill et al. [24] had one or more alterations previously suggested to confer resistance to an FDA-approved on-label therapy, which would also inform clinical decision-making.

Studies in HNSCC specifically evaluating the value of ctDNA for personalized treatment selection are lacking so far. The largest genetic landscape analysis of ctDNA was performed within the framework of the randomized multicenter phase II trial BERIL-1 in which the efficacy of buparlisib (BKM120), an oral pan-PI3K 
inhibitor plus paclitaxel or placebo plus paclitaxel was evaluated in patients with R/M HNSCC progressing on/after one previous platinum-based chemotherapy regimen for R/M disease [36]. In the accompanying biomarker study, ctDNA mutation profiles could be established in 112/158 patients (71\%) using targeted NGS [37]. The percentage of actionable alterations detected in liquid biopsies and the overall concordance with tumor tissue were not directly reported by the investigators. However, as derivable from the presented overview of the most frequent gene alterations in ctDNA at screening [37], alterations in genes of the PI3K/AKT/mTOR pathway (PIK3CA, PIK3CG, PIK3C2G, PIK3R1, PIK3R4, PIK3R5, AKT3, PTEN, RICTOR, RPTOR, TSC1, TSC2, MTOR) were found in 29/112 patients (26\%). This suggest that ctDNA mutation profiling could indeed be used to select patients with gene alterations druggable by inhibitors in clinical development for HNSCC. Per BERIL-1 protocol, the PI3K activation status was defined as the presence of a PIK3CA mutation and/or a loss of PTEN expression [37]. Statistical analyses did not suggest a difference in OS between the buparlisib and placebo arms in the PI3Kactivated subgroup, however, the low number of patients in this subgroup $(\mathrm{n}=18)$ weakened the statistical power to evaluate a possible relationship between genotype and clinical outcome [37].

Braig et al. were the first group to study processes of clonal tumor evolution occurring in HNSCC tumors under pressure of molecular therapy. Patients receiving cetuximab/platinum/5-fluorouracil treatment for R/M HNSCC were included in this prospective biomarker study. Targeted NGS was used for detection of mutations in four genes (EGFR, KRAS, NRAS and HRAS) in diagnostic tumor tissue as well as blood samples taken under and after completion of combination therapy/maintenance [38]. Mutations in the four genes were not detected in tumor tissue of cetuximab-naïve patients, except for HRAS mutations in $4.3 \%$ of patients. Interestingly, $46 \%$ of patients with on-treatment disease progression showed acquired $R A S$ mutations in ctDNA, while no $R A S$ mutations were found in the non-progressive subset of patients, indicating that acquisition of RAS mutant clones correlated significantly with clinical resistance [38]. Of note, the emergence of mutations preceded clinical progression in half of the patients, with a maximum time from mutation detection to clinical progression of 16 weeks [38]. These findings corroborate previous results from colon cancer where KRAS mutations were identified as frequent drivers of acquired resistance to cetuximab, and could be detected in blood of cetuximab-treated patients as early as 10 months before radiographic progression [39].

\section{Circulating Tumor Cells for Prognosis of Outcome in HNSCC}

Evidence of a potential role of CTCs in disease progression of HNSCC has been provided by numerous independent studies over the last 30 years. A review of these studies would be beyond the scope of this article. I would therefore like to refer the 
reader to two recent reviews on this topic [40, 41]. In our own study in locally advanced HNSCC patients treated with surgery and adjuvant chemoradiation [42], a significant negative association between the persistence of CTCs after surgery and outcome was observed. The use of different cut-offs for definition of CTC-positive blood samples and the inclusion of heterogeneously treated patient cohorts in the majority of previous studies at least call for caution with regard to a definite conclusion on the prognostic value of CTCs.

The availability of robust, easy-to-handle CTC detection devices such as the CellSearch ${ }^{\circledast}$ platform has opened the door for the integration of CTC analysis into clinical routine. However, the mere enumeration of CTCs has proven insufficiently informative to prompt widespread clinical adoption. There is accumulating evidence that more extended phenotyping of CTCs might be necessary for improving their diagnostic value. Identification of the true metastasis-inducing subclones within the bulk CTC population remains a challenge but is imperative in order to improve the diagnostic potential of CTCs. Genome-wide single-cell RNA-seq and DNA-seq performed in CTCs have already provided crucial new insights into CTC heterogeneity and mechanisms of therapeutic resistance in other cancer types [43, 44], but such analyses still have to be done in HNSCC. These analyses combined with multiparametric CTC phenotyping by imaging flow cytometry or automated immunofluorescence microscopy setups [41] will certainly increase our understanding on the relevant biological mechanisms endowing CTCs with the potential to emigrate from the primary site to blood circulation, to survive their journey and to re-seed at distant organs, thereby supporting the development of CTCs as liquid biomarker in HNSCC.

\section{Conclusions}

Liquid biopsies have been successfully used to guide treatment decisions in patients with lung cancer harboring EGFR and ALK mutations. This review summarized the current evidence from the literature pointing to a clinical potential in HNSCC as well (Table 4.2). Prospective randomized clinical studies are needed to firmly establish the usefulness of liquid biopsy for detecting molecular markers in clinical practice, by demonstrating that treatment decisions based on liquid biopsies result in better outcome. In addition, the persistence of CTCs after surgery should be studied further to determine whether they indicate the need for adjuvant therapy regardless of the tumor size or nodal status. Currently, one of the most promising use of liquid biopsies is in the detection of cancer progression and development of drug resistance. Liquid biopsies may help in elucidating the molecular resistance mechanisms in cetuximab-containing regimens. However, prospective evidence on the usefulness of liquid biopsies in the assessment of drug responses to achieve the best benefit for HNSCC patients is still needed. 
Table 4.2 Promising clinical applications for liquid biopsies in HNSCC: Evidence from the literature

\begin{tabular}{l|l|l|l|l}
\hline \multirow{2}{*}{ Aim } & Biomarker & Technique & $\begin{array}{l}\text { Tumor } \\
\text { localisation }\end{array}$ & References \\
\hline Early detection & $\begin{array}{l}\text { cfDNA methylation } \\
\text { pattern }\end{array}$ & $\begin{array}{l}\text { Targeted bisulfite } \\
\text { sequencing }\end{array}$ & All sites & {$[17,18]$} \\
\cline { 2 - 5 } & EBV DNA & $\begin{array}{l}\text { Quantitative } \\
\text { real-time PCR }\end{array}$ & NPC & {$[7]$} \\
\cline { 2 - 5 } & HPV DNA & Droplet PCR & HPV+ OPC & {$[8]$} \\
\hline $\begin{array}{l}\text { Posttreatment } \\
\text { surveillance }\end{array}$ & HPV DNA & Droplet PCR & HPV+ OPC & {$[9,10]$} \\
\cline { 2 - 5 } & TP53 mutations & Targeted NGS & $\begin{array}{l}\text { HPV- } \\
\text { HNSCC }\end{array}$ & {$[14]$} \\
\cline { 2 - 5 } & CTCs & $\begin{array}{l}\text { Imaging flow } \\
\text { cytometry, qPCR }\end{array}$ & All sites & {$[40-42]$} \\
\hline $\begin{array}{l}\text { Molecular treatment } \\
\text { selection }\end{array}$ & $\begin{array}{l}\text { Actionable } \\
\text { alterations }\end{array}$ & Targeted NGS & All sites & $\begin{array}{l}{[24,27,} \\
36]\end{array}$ \\
\hline Response monitoring & $\begin{array}{l}\text { HRAS, KRAS, NRAS } \\
\text { mutations }\end{array}$ & Targeted NGS & R/M HNSCC & {$[38]$} \\
\hline
\end{tabular}

cfDNA cell-free deoxynucleic acid, CTCs circulating tumor cells, EBV Epstein-Barr virus, HNSCC head neck squamous cell carcinoma, $H P V$ human papillomavirus, $N G S$ next-generation sequencing, $N P C$ nasopharyngeal carcinomas, $P C R$ polymerase chain reaction, $R / M$ recurrent/metastatis

\section{References}

1. Diamantis A, Magiorkinis E, Koutselini H. Fine-needle aspiration (FNA) biopsy: historical aspects. Folia Histochem Cytobiol. 2009;47(2):191-7.

2. Oxnard GR, Thress KS, Alden RS, et al. Association between plasma genotyping and outcomes of treatment with Osimertinib (AZD9291) in advanced non-small-cell lung cancer. J Clin Oncol. 2016;34(28):3375-82.

3. Oxnard GR, Paweletz CP, Kuang Y, et al. Noninvasive detection of response and resistance in EGFR-mutant lung cancer using quantitative next-generation genotyping of cell-free plasma DNA. Clin Cancer Res. 2014;20(6):1698-705.

4. Garcia-Murillas I, Chopra N, Comino-Méndez I, et al. Assessment of molecular relapse detection in early-stage breast cancer. JAMA Oncol. 2019;5(10):1473-8.

5. Lo YM, Chan LY, Lo KW, et al. Quantitative analysis of cell-free Epstein-Barr virus DNA in plasma of patients with nasopharyngeal carcinoma. Cancer Res. 1999;59(6):1188-91.

6. Chan KC, Zhang J, Chan AT, et al. Molecular characterization of circulating EBV DNA in the plasma of nasopharyngeal carcinoma and lymphoma patients. Cancer Res. 2003;63(9):2028-32.

7. Chan KCA, Woo JKS, King A, et al. Analysis of plasma Epstein-Barr virus DNA to screen for nasopharyngeal cancer. N Engl J Med. 2017;377(6):513-22.

8. Damerla RR, Lee NY, You D, et al. Detection of early human papillomavirus-associated cancers by liquid biopsy. JCO Precis Oncol 2019;3.

9. Chera BS, Kumar S, Shen C, et al. Plasma circulating tumor HPV DNA for the surveillance of cancer recurrence in HPV-associated oropharyngeal cancer. J Clin Oncol. 2020;38(10):1050-8.

10. Chera BS, Kumar S, Beaty BT, et al. Rapid clearance profile of plasma circulating tumor HPV type 16 DNA during chemoradiotherapy correlates with disease control in HPV-associated oropharyngeal cancer. Clin Cancer Res. 2019;25(15):4682-90.

11. The Cancer Genome Atlas network. Comprehensive genomic characterization of head and neck squamous cell carcinomas. Nature. 2015;517(7536):576-82. 
12. Poeta ML, Manola J, Goldenberg D, et al. The Ligamp TP53 assay for detection of minimal residual disease in head and neck squamous cell carcinoma surgical margins. Clin Cancer Res. 2009;15(24):7658-65.

13. Huang X, Pateromichelakis S, Hills A, et al. p53 mutations in deep tissues are more strongly associated with recurrence than mutation-positive mucosal margins. Clin Cancer Res. 2007;13(20):6099-106.

14. van Ginkel JH, de Leng WW, de Bree R, et al. Targeted sequencing reveals TP53 as a potential diagnostic biomarker in the post-treatment surveillance of head and neck cancer. Oncotarget. 2016;7(38):61575-86.

15. Xie M, Lu C, Wang J, et al. Age-related mutations associated with clonal hematopoietic expansion and malignancies. Nat Med. 2014;20(12):1472-8.

16. Leary RJ, Sausen M, Kinde I, et al. Detection of chromosomal alterations in the circulation of cancer patients with whole-genome sequencing. Sci Transl Med. 2012;4(162):162ra54.

17. https://grail.com/clinical-studies/circulating-cell-free-genome-atlas-study/

18. Liu MC, Oxnard GR, Klein EA, et al. Sensitive and specific multi-cancer detection and localization using methylation signatures in cell-free DNA. Ann Oncol. 2020;31(6):745-59.

19. Alexandrov LB, Nik-Zainal S, Wedge DC, et al. Signatures of mutational processes in human cancer. Nature. 2013;500(7463):415-21.

20. Zhang XC, Xu C, Mitchell RM, et al. Tumor evolution and intratumor heterogeneity of an oropharyngeal squamous cell carcinoma revealed by whole-genome sequencing. Neoplasia. 2013;15(12):1371-8.

21. Ledgerwood LG, Kumar D, Eterovic AK, et al. The degree of intratumor mutational heterogeneity varies by primary tumor sub-site. Oncotarget. 2016;7(19):27185-98.

22. Tabatabaeifar S, Thomassen M, Larsen MJ, et al. The subclonal structure and genomic evolution of oral squamous cell carcinoma revealed by ultra-deep sequencing. Oncotarget. 2017;8(10):16571-80.

23. Bettegowda C, Sausen M, Leary RJ, et al. Detection of circulating tumor DNA in early- and late-stage human malignancies. Sci Transl Med. 2014;6(224):224ra24.

24. Zill OA, Banks KC, Fairclough SR, et al. The landscape of actionable genomic alterations in cell-free circulating tumor DNA from 21,807 advanced cancer patients. Clin Cancer Res. 2018;24(15):3528-38.

25. Schwaederle M, Chattopadhyay R, Kato S, et al. Genomic alterations in circulating tumor DNA from diverse cancer patients identified by next-generation sequencing. Cancer Res. 2017;77(19):5419-27.

26. Wang Y, Springer S, Mulvey CL, et al. Detection of somatic mutations and HPV in the saliva and plasma of patients with head and neck squamous cell carcinomas. Sci Transl Med. 2015;7(293):293ra104.

27. Galot R, van Marcke C, Helaers R, et al. Liquid biopsy for mutational profiling of locoregional recurrent and/or metastatic head and neck squamous cell carcinoma. Oral Oncol. 2020;104:104631.

28. Perdomo S, Avogbe PH, Foll M, et al. Circulating tumor DNA detection in head and neck cancer: evaluation of two different detection approaches. Oncotarget. 2017;8(42):72621-32.

29. Froyen G, Broekmans A, Hillen F, et al. Validation and application of a custom-designed targeted next-generation sequencing panel for the diagnostic mutational profiling of solid tumors. PLoS One. 2016;11(4):e0154038.

30. Kurtz DM, Esfahani MS, Scherer F, et al. Dynamic risk profiling using serial tumor biomarkers for personalized outcome prediction. Cell. 2019;178(3):699-713.e19.

31. Scherer F, Kurtz DM, Newman AM, et al. Distinct biological subtypes and patterns of genome evolution in lymphoma revealed by circulating tumor DNA. Sci Transl Med. 2016;8(364):364ra155.

32. Newman AM, Lovejoy AF, Klass DM, et al. Integrated digital error suppression for improved detection of circulating tumor DNA. Nat Biotechnol. 2016;34(5):547-55. 
33. Maheswaran S, Sequist LV, Nagrath S, et al. Detection of mutations in EGFR in circulating lung-cancer cells. N Engl J Med. 2008;359(4):366-77.

34. Tzanikou E, Markou A, Politaki E, et al. PIK3CA hotspot mutations in circulating tumor cells and paired circulating tumor DNA in breast cancer: a direct comparison study. Mol Oncol. 2019;13(12):2515-30.

35. Kidess-Sigal E, Liu HE, Triboulet MM, et al. Enumeration and targeted analysis of KRAS, BRAF and PIK3CA mutations in CTCs captured by a label-free platform: comparison to ctDNA and tissue in metastatic colorectal cancer. Oncotarget. 2016;7(51):85349-64.

36. Soulières D, Faivre S, Mesía R, et al. Buparlisib and paclitaxel in patients with platinumpretreated recurrent or metastatic squamous cell carcinoma of the head and neck (BERIL-1): a randomised, double-blind, placebo-controlled phase 2 trial. Lancet Oncol. 2017;18(3):323-35.

37. Soulières D, Licitra L, Mesía R, et al. Molecular alterations and Buparlisib efficacy in patients with squamous cell carcinoma of the head and neck: biomarker analysis from BERIL-1. Clin Cancer Res. 2018;24(11):2505-16.

38. Braig F, Voigtlaender M, Schieferdecker A, et al. Liquid biopsy monitoring uncovers acquired RAS-mediated resistance to cetuximab in a substantial proportion of patients with head and neck squamous cell carcinoma. Oncotarget. 2016;7(28):42988-95.

39. Misale S, Yaeger R, Hobor S, et al. Emergence of KRAS mutations and acquired resistance to anti-EGFR therapy in colorectal cancer. Nature. 2012;486(7404):532-6.

40. Economopoulou P, Kotsantis I, Kyrodimos E, et al. Liquid biopsy: an emerging prognostic and predictive tool in head and neck squamous cell carcinoma (HNSCC). Focus on circulating tumor cells (CTCs). Oral Oncol. 2017;74:83-9.

41. Tinhofer I, Staudte S. Circulating tumor cells as biomarkers in head and neck cancer: recent advances and future outlook. Expert Rev Mol Diagn. 2018;18(10):897-906.

42. Tinhofer I, Konschak R, Stromberger C, et al. Detection of circulating tumor cells for prediction of recurrence after adjuvant chemoradiation in locally advanced squamous cell carcinoma of the head and neck. Ann Oncol. 2014;25(10):2042-7.

43. Aceto N, Bardia A, Miyamoto DT, et al. Circulating tumor cell clusters are oligoclonal precursors of breast cancer metastasis. Cell. 2014;158(5):1110-22.

44. Yu M, Ting DT, Stott SL, et al. RNA sequencing of pancreatic circulating tumour cells implicates WNT signalling in metastasis. Nature. 2012;487(7408):510-3.

Open Access This chapter is licensed under the terms of the Creative Commons Attribution 4.0 International License (http://creativecommons.org/licenses/by/4.0/), which permits use, sharing, adaptation, distribution and reproduction in any medium or format, as long as you give appropriate credit to the original author(s) and the source, provide a link to the Creative Commons license and indicate if changes were made.

The images or other third party material in this chapter are included in the chapter's Creative Commons license, unless indicated otherwise in a credit line to the material. If material is not included in the chapter's Creative Commons license and your intended use is not permitted by statutory regulation or exceeds the permitted use, you will need to obtain permission directly from the copyright holder.

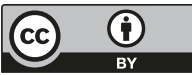

